\title{
Measurement of bone mineral in vivo: An improved method*
}

John R. Cameron, ${ }^{(1)}$ James Sorenson. (I)

\begin{abstract}
The mineral content of bone can be determined by measuring the absorption by bone of a monochromatic, low-energy photon beam which originates in a radioactive source (iodine-I 25 at $27.3 \mathrm{kev}$ or americium-24I at $59.6 \mathrm{kev}$ ). The intensity of the beam transmitted by the bone is measured by counting with a scintillation detector. Since the photon source and detector are well collimated, errors resulting from scattered radiation are reduced. From measurements of the intensity of the transmitted beam, made at intervals across the bone, the total mineral content of the bone can be determined. The results are accurate and reproducible to within about 3 percent.
\end{abstract}

A $\mathrm{n}$ improved method for measuring the mineral content of bone in vivo by photon absorption techniques has been developed. The methods previously described in the literature ${ }^{1,2}$ are based on measurement of the transmission through bone of photon beams which are generated by standard $\mathrm{x}$-ray tubes. The transmission is usually determined by densitometric measurements of $\mathrm{x}$-ray films. The method described here differs from each of these earlier methods in one or more ways: in this method (i) the transmission of the photon beam is measured directly by counting techniques, by means of a scintillation detector system; (ii) the photon beam used is essentially monochromatic; (iii) the photon beam and detector are well collimated; and (iv) the effects of the tissue around the bone are taken into account. These factors eliminate errors resulting from the variability of x-ray films and film development techniques, reduce uncertainties in absorption coefficients, reduce the effects of scattered radiation, and reduce errors arising from the presence of tissue.

Figure 1 is a schematic diagram of the equipment used. The radioactive photon source used at present is iodine- $125(5 \mathrm{mc})$ contained in a thin-walled stainless steel tube $1 \mathrm{~cm}$ long, $3 \mathrm{~mm}$ in diameter. [An americium-241 source $(1 \mathrm{mc})$ in tubing of similar dimensions is also used.] The tube is placed in a hole $3 \mathrm{~mm}$ in diameter in a small lead cube. The hole is drilled in such a way that the end of the tube is $5 \mathrm{~mm}$ below the surface of the lead, for purposes of collimating the photon beam. The tube is viewed end-on by the crystal detector system, which is also collimated, as shown schematically in Fig. 1. Two holes, each $3 \mathrm{~mm}$ in diameter, drilled in a pair of lead plates, each 5 $\mathrm{mm}$ thick, serve as collimating apertures. The plates are $4 \mathrm{~cm}$ apart, and the two holes are aligned on a common axis with the photon source.

The source and the detector system are rigidly coupled by mechanical means and are driven simultaneously in 1-mm steps, in a direction transverse to the bone, by the motor-drive system. Measurements of the transmission of the photon beam through the bone are made for a 10 -second

(I) Departments of Radiology and Physics, University of Wisconsin, Madison.

From: Cameron JR, Sorenson J. Measurement of bone mineral in vivo: An improved method. Science 1963;142:230-232. Reprinted with kind permission from the American Association for the Advancement of Science. 


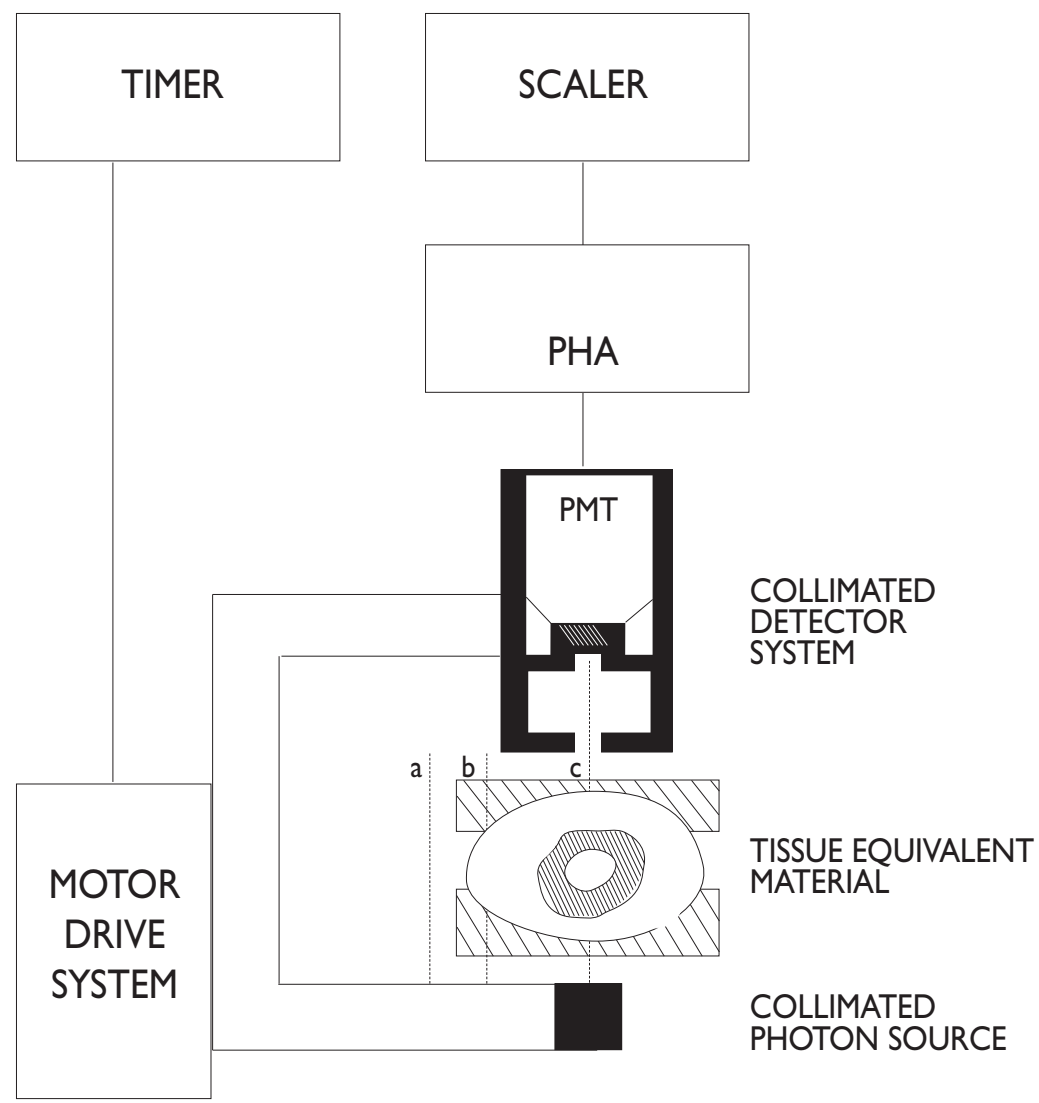

Fig. I. Schematic diagram OF EQUiPMENT FOR MEASURING THE MINERAL CONTENT OF BONE

interval after each step. The starting and stopping of the drive motor and scaler and the resetting of the scaler are automatically performed by the timer unit, and the operator need only record the scaler reading after each step. In the course of the scan, measurements of the transmission of the photon beam through tissue alone, on either side of the bone, are also made. From the data obtained in these measurements, an equivalent thickness of bone mineral can be computed, as follows.

Let $I_{0}$ be the intensity of the unobstructed photon beam, (as measured at $a$ of Fig. 1); $I_{0}^{*}$ the intensity after passage of the beam through a thickness $T$ of tissue, (as measured through $b$ of Fig. 1 ); and $I$ the intensity after passage of the beam through an equal thickness of bone mineral plus tissue $T_{\mathrm{b}}+T_{\mathrm{m}}$ (as measured through $c$ of Fig. 1 ). If $\mu_{\mathrm{b}}$ and $\mu_{\mathrm{m}}$ are the mass absorption coefficients of bone mineral and of tissue, respectively, then:

$$
\begin{aligned}
I_{o}^{*} & =I_{o} \exp \left(-\mu_{\mathrm{m}} \rho_{\mathrm{m}} T\right) \\
I & =I_{o} \exp \left(-\mu_{\mathrm{m}} \rho_{\mathrm{m}} T_{\mathrm{m}}-\mu_{\mathrm{b}} \rho_{\mathrm{b}} T_{\mathrm{b}}\right) \\
& =I_{o} \exp \left(-\mu_{\mathrm{m}} \rho_{\mathrm{m}}\left(T-T_{\mathrm{b}}\right)-\mu_{\mathrm{b}} \rho_{\mathrm{b}} T_{\mathrm{b}}\right) \\
& =I_{o} \exp \left(-\mu_{\mathrm{m}} \rho_{\mathrm{m}} T\right) \exp \left(-\mu_{\mathrm{b}} \rho_{\mathrm{b}} T_{\mathrm{b}}+\mu_{\mathrm{m}} \rho_{\mathrm{m}} T_{\mathrm{b}}\right) \\
I & =I_{o}^{*} \exp \left(-\mu_{\mathrm{b}} \rho_{\mathrm{b}} T_{\mathrm{b}}+\mu_{\mathrm{m}} \rho_{\mathrm{m}} T_{\mathrm{b}}\right) \\
T_{\mathrm{b}} & =\left[\log _{\mathrm{e}}\left(I_{o}^{*} / I\right)\right] /\left(\mu_{\mathrm{b}} \rho_{\mathrm{b}}-\mu_{\mathrm{m}} \rho_{\mathrm{m}}\right)
\end{aligned}
$$

Equation 1 gives an equivalent thickness of compact bone mineral of density $\rho_{b}$ for the point at which the intensity of the transmitted photon beam is I. Measuring I at closely spaced intervals 
across the bone gives a series of equivalent thicknesses which, when summed, give the equivalent cross-sectional area of compact bone mineral in the bone. A standard composition of bone mineral is assumed in these calculations. ${ }^{3}$ The absorption coefficient $\mu_{\mathrm{b}}$ can be determined on the basis of this assumption from tabulated atomic absorption coefficients. ${ }^{4}$ It is also assumed that all non-bonemineral substances absorb radiation to the degree that striated muscle tissue does. The absorption coefficient $\mu_{\mathrm{m}}$ can be calculated in a similar manner, or it can be experimentally determined for the particular tissue under study.

As shown in Fig. 1, the bone and tissue under observation are placed between form-fitting pieces of tissue equivalent material [for example, Mix-D ${ }^{5}$ with parallel opposite faces. This is done to make sure that the quantities $I_{0}{ }^{*}$ and I are measured for equal thicknesses of "tissue" or of tissue plus bone; equal thicknesses are necessary if the mathematical relationships given in Eq. 1 are to be true.

The method has been used to determine the mineral content of bone in over 200 persons. At present, the 10-second transmission counts are recorded from the scaler at each of the 1-mm intervals across the bone. These transmission measurements are plotted at equal intervals on semilogarithmic graph paper, and a smooth curve is drawn through them. The extended $I_{0}^{*}$ curve is also drawn in, as though the bone were replaced with tissue. Examples of such graphs are shown in Fig. 2; these are graphs for scans, made with $\mathrm{I}^{125}$ as a radiation source, on a typical normal female and a typical

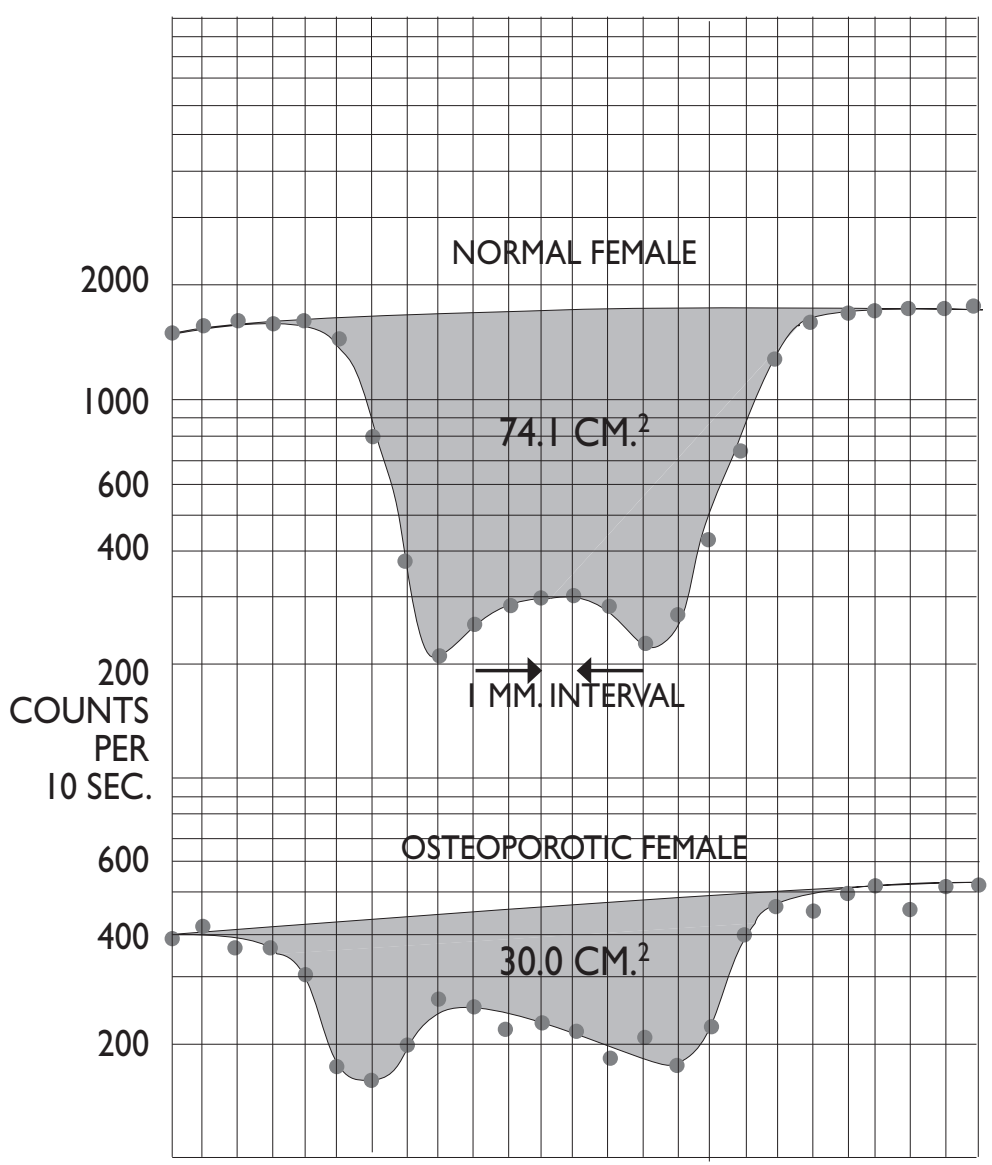

Fig. 2. SCANNING RECORDS FOR A 28-YEAR-OLD NORMAL FEMALE AND FOR A 75-YEAR-OLD FEMALE WITH OSTEOPOROSIS 
osteoporotic female. The distance between the two curves at any point is directly proportional to $\log _{\mathrm{e}}\left(I_{0}^{*} / I\right)$, and hence, in accordance with Eq. 1, to $T_{\mathrm{b}}$. The total area between the curves, which can be accurately measured with a planimeter, is thus proportional to the cross-sectional area of bone mineral in the path scanned. This area of bone mineral is numerically equal to the volume of bone mineral per unit length of bone. From the known density ${ }^{2}$ one can determine the mass of bone mineral in a unit length of bone.

The scans recorded in Fig. 2 were made on the radius of the left arm, about $10 \mathrm{~cm}$ from the distal end. Figure 3 is a plot of the mineral content of bone relative to age for 137 female subjects; the scans were made on the left radius. The content of bone mineral is given in grams of hydroxyapatite per 1-cm length of bone. Data points for patients for whom a clinical diagnosis of osteoporosis had been made are indicated by a star. The phenomenon of "postmenopausal osteoporosis" after age 50 is clearly noticeable.

Repeat measurements have been made for most subjects, and in general, results have been found to be reproducible to within 3 percent. A common source of error in the reproducibility data is movement on the part of the subject while the scan is in progress.

This error appears in the results as a change in the apparent width of the bone. The measured area can be adjusted to minimize this error by comparing the apparent width as determined from the scan with the width measured from an $x$-ray of the bone. The results of this method of determining the content of bone mineral are accurate to within about 3 percent, as determined by studies on bone phantoms.

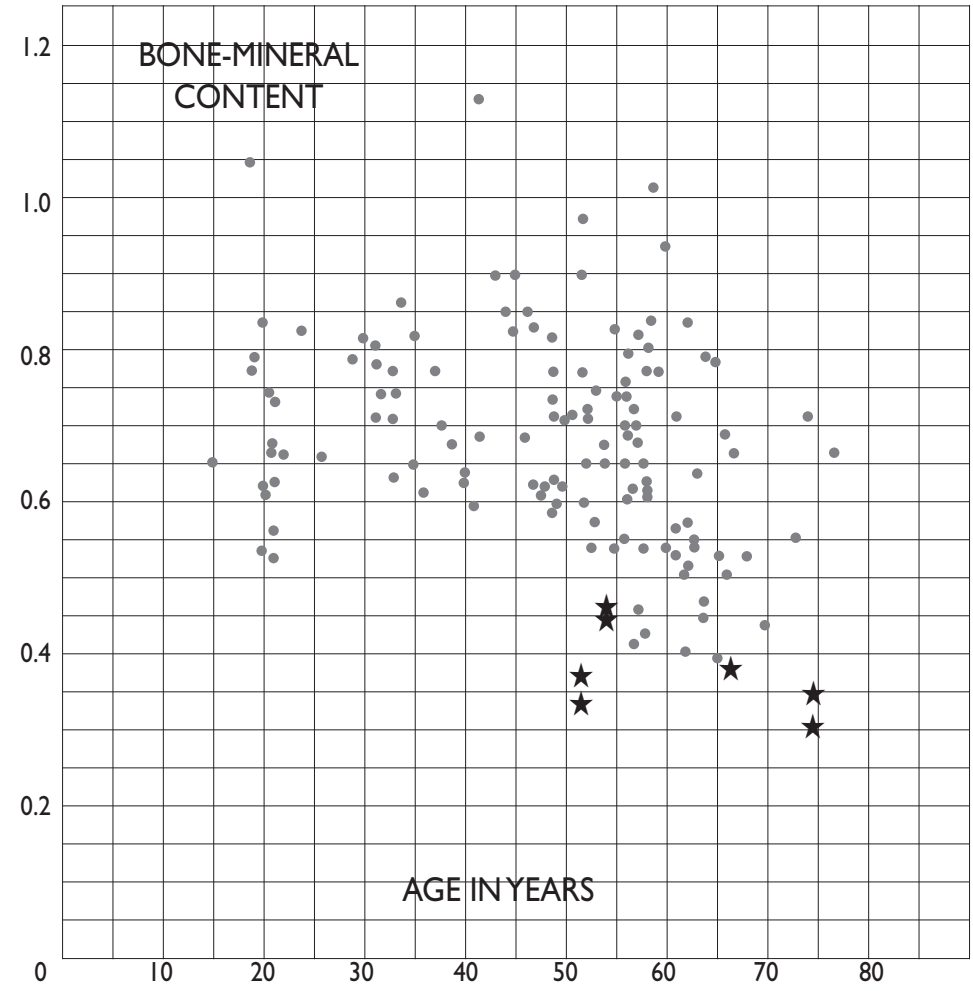

Fig. 3. Plot of mineral content of bone versus age distribution for I 37 female subjects. The MINERAL CONTENT IS GIVEN IN GRAMS OF CALCIUM HYDROXYAPATITE PER CENTIMETER LENGTH OF BONE 
It is possible to extend this method to obtain information about the composition of the bone mineral. The mass absorption coefficient of a homogenous combination of $n$ elements, such as bone mineral, can be expressed as

$$
\mu_{\mathrm{b}}=f_{1} \mu_{1}+f_{2} \mu_{2}+\ldots+f_{\mathrm{n}} \mu_{\mathrm{n}}
$$

where $f_{1}, f_{2}, \ldots f_{\mathrm{n}}$ are the fractions (by weight) of the elements present and $\mu_{1}, \mu_{2} \ldots \mu_{\mathrm{n}}$ are the mass absorption coefficients of these elements. These absorption coefficients are known, ${ }^{4}$ so that when this expression for $\mu_{\mathrm{b}}$ is substituted in Eq. 1 , an equation in $n+1$ unknowns results, $f_{1^{\prime}} f_{2^{\prime}} \ldots f_{\mathrm{n}^{\prime}}$ and $T_{\mathrm{b}}$. If $I_{0}^{*} / I$ is measured at $n$ different photon energies, a set of $n$ equations of the form of Eq. 1 is obtained. These $n$ equations, along with the equation

$$
f_{1}+f_{2}+\ldots+f_{\mathrm{n}}=1 \text {, }
$$

form a system of $n+1$ equations in $n+1$ unknowns which may be solved by algebraic methods for $f_{1}, f_{2} \ldots f_{\mathrm{n}}$ and $T_{\mathrm{b}}$. The primary mineral elements of bone are calcium and phosphorus, so, in principle, the $\mathrm{Ca} / \mathrm{P}$ ratio for a particular bone could be determined by measuring $I_{0}^{*} / I$ over the same point on the bone at two different photon energies.

Equation 1 may be transformed to read

$$
A_{\mathrm{b}}=C \times G /\left(\mu_{\mathrm{b}} \rho_{\mathrm{b}}-\mu_{\mathrm{m}} \rho_{\mathrm{m}}\right)
$$

where $A_{\mathrm{b}}$ is the amount of bone mineral in a cross section of bone and $G$ is the graph area obtained by plotting and measuring the area between the $I$ and $I_{0}^{*}$ curves on semilogarithmic graph paper, as described previously. $C$ is a constant which depends on the physical dimensions of the graph paper used.

If $G$ is measured at two different energies, the $\mathrm{Ca} / \mathrm{P}$ ratio for the cross section of bone under observation could be obtained from Eq. 3 and two equations of the form of Eq. 4. This method could also be used to determine the relative amount of fat in tissue.

This technique has been tested on a two-phase material with ${ }^{125}$ (27.3 kev) and $\mathrm{Am}^{241}$ (59.6 $\mathrm{kev}$ ) as radioactive sources. The materials used were blocks of paraffin and $\mathrm{CaCO}_{3}$, homogeneously mixed in known proportions. The measurements resulted in determination of the percentage, by weight, of $\mathrm{CaCO}_{3}$ present in the blocks, over a wide range of compositions, to an average accuracy of within 3 percent.

The principal errors at the present time are the result of uncertainties in the absorption coefficients and the lack of complete monochromaticity in the photon sources. These errors can be reduced through further study. If the only errors involved were statistical ones, an accuracy to within less than 2 percent could be expected in determining the mineral content of bone by this method.

The radiation exposure per scan with the techniques described is of the order of 0.15 rem with an $\mathrm{I}^{125}(27.3 \mathrm{kev})$ source of 5-mc activity. The exposure is about $0.05 \mathrm{rem}$ when a source of $\mathrm{Am}^{241}(59.6$ $\mathrm{kev}$ ) of 5-mc activity is used. This exposure is limited to a small area of the forearm and should be compared with the maximum permissible dose to the forearms of children of 7.5 rem per year. ${ }^{6,7}$

Note added in proof. We have found a simple method for making "point" sources of $\mathrm{I}^{125}$. Iodine is removed from solutions by an ion-exchange resin, Dowex $1 \times 4,20-50$ mesh. ${ }^{8}$ Single grains of the resin, allowed to stand for periods of about 48 hours in freshly prepared carrier-free radioactive iodine solutions, will take up $5 \mathrm{mc}$ of iodine. Grain diameters are less than 1 millimeter. 


\section{References and Notes}

I. E. H. Mayer et al., Radiation Res. I3, 156 (1960); D. E.Williams and R. L. Mason, Science 138, 39 (I962).

2. K.A. Omnell,Acta Radiol. Suppl. 1957, No. 148 (1957).

3. In the standard composition, calcium hydroxyapatite is the basic constituent. For a comprehensive review of work on this subject see K.A. Omnell (2).

4. J.A.Victoreen, J.Appl. Phys. 20, II4I (1949).

5. D. E.A. Jones and H. C. Raine, Brit. J. Radiol. 22, 549 (1949). The composition of the Mix-D used in our work is as follows: 55.2 percent (by weight) paraffin, 27.6 percent polyethylene, I2.5 percent $\mathrm{MgO}, 4.7$ percent TiO2. It has been experimentally determined that Mix-D of this composition corresponds best with tissue of the forearm with respect to absorption of radiation.

6. Federal Register 25, 104 (17 Nov. 1960).

7. We thank Dr. F. C. Larsen for his interest and advice, and David Knutsen, John Duffy, and Mrs. Susan Steinhart for assistance in making measurements and handling data. This work was supported in part by the Wisconsin Alumni Research Foundation; by the James Picker Foundation, on recommendation of the Committee on Radiology, National Academy of Sciences-National Research Council; and by the National Institutes of Health.

8. J.T. Baker Chemical Co., Phillipsburg, N.J. 29 July 1963 